\title{
New energy-spread-feedback control system using nondestructive energy-spread monitors
}

\author{
T. Suwada, ${ }^{*}$ M. Satoh, and K. Furukawa \\ Accelerator Laboratory, High Energy Accelerator Research Organization (KEK), 1-1 Oho, Tsukuba, Ibaraki 305-0801, Japan
}

(Received 30 June 2005; published 23 November 2005)

\begin{abstract}
A new energy-spread feedback control system using nondestructive energy-spread monitors has been developed in order to control and stabilize the energy spreads of single-bunch electron and positron beams in the KEKB injector linac. A well-controlled feedback system has been successfully working in daily operation not only for keeping the injection rate higher along with the beam-orbit and energy-feedback control system, but also for reducing the background level to the KEKB collider experiment. The energy spreads of the injection beams have been well stabilized within $0.33 \pm 0.01 \%$ and $0.50 \pm 0.02 \%(0.55 \pm$ $0.06 \%$ ) for the electron beam and the first (second) bunch of the high-current primary electron beam for positron production at the $180^{\circ}$ arc, respectively, and within $0.39 \pm 0.01 \%(0.36 \pm 0.03 \%)$ for the first (second) bunch of the positron beam at the end of the injector linac through the energy-spread feedback control system under the nominal operation condition. In this report we describe in detail the energyspread feedback control system using nondestructive energy-spread monitors with multi-strip-line electrodes and their performance in KEKB operation.
\end{abstract}

DOI: 10.1103/PhysRevSTAB.8.112802

PACS numbers: 29.17.+w, 07.05.Hd, 41.75.Ht, 41.85.Qg

\section{INTRODUCTION}

It is of fundamental importance to control and stabilize the acceleration and transport of bunched beams without any instabilities in high-energy accelerators in order to increase the luminosity in a collider experiment. It is strongly required for so-called factory colliding machines to attain not only higher peak luminosities, but also higher integrated luminosities in order to reduce the statistical errors to be as small as possible in physics analysis. For this purpose, well-controlled accelerator operation should be accomplished by utilizing a dedicated beam-feedback control system along with a sophisticated beam diagnostic and monitoring system, which can make precise measurements of the dynamical beam parameters.

The KEK B-Factory (KEKB) project [1] is in progress for testing $\mathrm{CP}$ violation in the decay of $\mathrm{B}$ mesons. KEKB is an asymmetric electron-positron collider comprising $3.5 \mathrm{GeV}$ positron and $8 \mathrm{GeV}$ electron rings. The KEKB injector linac [2] injects single-bunch positron and electron beams directly into the KEKB storage rings. The beam charges are designed to be 0.64 and $1.3 \mathrm{nC} /$ bunch for the positron and electron beam, respectively. A high-current primary electron beam $(\sim 10 \mathrm{nC} /$ bunch $)$ is required in order to generate sufficient positrons. Since the KEKB is a factory machine, well-controlled operation of the injector linac is strongly required for keeping the injection rate as high as possible and for maintaining the stability of beam collisions.

For this purpose, several beam-feedback control systems using dedicated beam diagnostic and monitoring tools are stably working in daily operation of the injector linac.

*Electronic address: tsuyoshi.suwada@kek.jp.
The beam-orbit feedback control system [3] with stripline-type beam-position monitors (BPMs) [4] controls to keep the transverse beam orbits stable; in particular, the transverse beam orbits of the high-current primary electron beam for positron production must be controlled in order to suppress any transverse wakefields. On the other hand, the beam-energy-feedback control system stabilizes the beam energies using BPMs installed at a large energy-dispersion section; in particular, the beam-energy feedback makes quick beam stabilization possible for a different beammode switch. These two kinds of feedback control systems have successfully stabilized the simultaneous acceleration and injection of two bunches separated by $96 \mathrm{~ns}$ in a rf pulse [5] for the positron beam. The two-bunch injection scheme has enabled us to boost the injection rate of the positron beam by a factor of 2 .

While these feedback control systems contribute to keep stable operation of the injector linac, it is also important to stabilize the energy spread in order not only to keep the injection rate higher, but also to reduce the background level to the detector and the radiation damage of the accelerator components. Off-energy particles in a bunch with a large energy spread cause the detector background; in particular, the longitudinal energy tail in a bunch not only reduces the injection rate, but also deteriorates the vacuum pressure at the injection point. Furthermore, the harder radiation damages the vacuum components around the injection point. The dedicated energy-spread feedback control system with nondestructive energy-spread monitors (ESMs) helps to cure the energy spread of the beams; in particular, this system is also expected to control stable acceleration and transport of the high-current primary electron beam in order to produce a higher amount of positrons. 


\section{NONDESTRUCTIVE ENERGY-SPREAD MONITOR SYSTEM}

\section{A. Detection principle}

Nondestructive ESMs using multi-strip-line electrodes have been newly installed in order to measure the energy spreads of $8-\mathrm{GeV}$ single-bunch electron and $3.5-\mathrm{GeV}$ positron beams delivered from the injector linac. The authors demonstrated that conventional strip-line-type monitors could nondestructively measure the spatial beam sizes of a single-bunch electron beam in the transverse directions with good accuracy [6]. The ESM is one of the applications for the beam-size measuring instrumentation, because the energy spread of a bunched beam is transformed to the transverse spatial spread at an energy-dispersion section. The detection principle and its performance have been reported in detail previously [7]. We provide a brief review here.

When a relativistic charged particle beam passes through a conducting vacuum pipe, induced image charges are simultaneously carried on the inner surface of the vacuum pipe along with the beam. A multipole-moment analysis [7] derives the second-order moment of the relativistic charged particle beam (termed "quadrupole moment") based on an analysis of the image-charge distribution along the azimuthal direction of the ESM, depending on the transverse beam positions and beam sizes. The quadrupole moment $\left(J_{q}\right)$ of the charged beam is simply formulated by

$$
J_{q}=\frac{1}{R^{2}}\left(\left\langle x^{2}\right\rangle-\left\langle y^{2}\right\rangle+\langle x\rangle^{2}-\langle y\rangle^{2}\right),
$$

where $R$ is the pipe radius of the ESM, $\langle x\rangle$ and $\langle y\rangle$ are the charge center of gravity of the beam, and $\left\langle x^{2}\right\rangle$ and $\left\langle y^{2}\right\rangle$ are the horizontal and vertical mean square half sizes of the beam, respectively. As shown from this formula, the quadrupole moment gives transverse beam-size information along with the transverse beam positions at the least orders. The quadrupole moment is also represented by using the pickup voltages $\left[V_{i}(i=1-N)\right]$ from multi-strip-line electrodes,

$$
J_{q}=\frac{\sum_{i=1}^{N} V_{i} \cos 2 \theta}{\sum_{i=1}^{N} V_{i}}
$$

Here, normalization by summing the $N$-pickup-signal voltages needs to cancel out the beam-charge fluctuation due to any beam measurement jitter. Since the transverse beam sizes are related to the detected pickup voltages through the quadrupole moment of the beam, they can be derived using these formula [Eqs. (1) and (2)] after correcting the transverse beam positions.

Assuming that the vertical energy dispersion $\left(\eta_{y}\right)$ is zero at the ESM, the energy spread $(\Delta E / E)$ of the beam can be estimated from the transverse beam sizes along with the optics parameters ( $\beta$ functions and horizontal dispersion $\left.\eta_{x}\right)$ and the transverse emittances $\left(\epsilon_{x}\right.$ and $\left.\epsilon_{y}\right)$, as follows:

$$
\left\langle x^{2}\right\rangle-\left\langle y^{2}\right\rangle \simeq \beta_{x} \epsilon_{x}+\left(\eta_{x} \Delta E / E\right)^{2}-\beta_{y} \epsilon_{y}+g,
$$

where $E$ is the beam energy and $g$ is the offset parameter, depending on the gain imbalance of the detection system and the alignment errors of the ESM. The offset parameter should be corrected based on a beam-based calibration. Thus, the ESM can measure nondestructively the energy spread through the quadrupole moment of the beam at an energy-dispersion section.

\section{B. Hardware design}

Figure 1 shows a schematic cross-sectional drawing of the ESM. The detailed mechanical design of the structure has been reported previously [7], and here, it is briefly summarized. The ESM is a conventional strip-line-type monitor with eight electrodes fabricated from stainless steel (SUS304) with $\pi / 4$ rotational symmetry. The stripline length $(L=1750 \mathrm{~mm})$ was determined to be as long as could possibly be installed into the limited spaces in the beam line so as to increase the signal-to-noise ratio. The pipe radius $(R=23.4 \mathrm{~mm})$ and the angular width $(\alpha=$ $15^{\circ}$ ) of the electrode were chosen so as to comprise a $50-\Omega$-transmission line. Eight pickups with a relatively narrow angular width are mounted with a tilt of $\pi / 8$ radian at the symmetrical polar coordinates in order to avoid any direct impinging of synchrotron radiation and off-energy electrons to the electrode surfaces in a large energydispersion section. A $50-\Omega$-vacuum feedthrough is connected to the upstream side of each electrode, while the downstream end is short-circuited to a vacuum duct in order to simplify the mechanical manufacturing process.

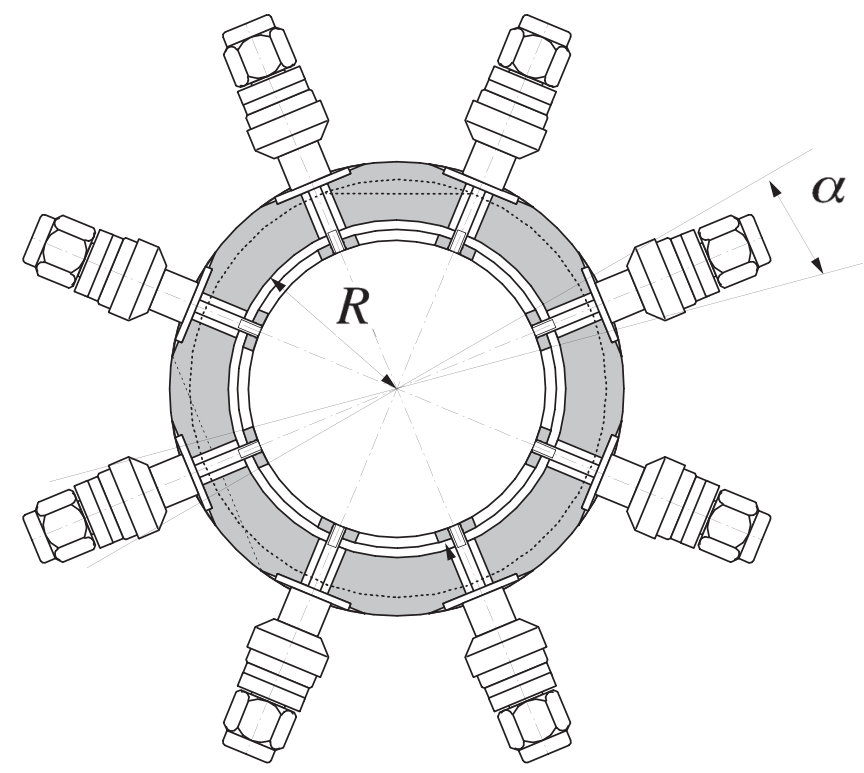

FIG. 1. Schematic cross-sectional drawing of the energyspread monitor. 


\section{Data-acquisition system}

Figure 2 shows a block diagram of the data acquisition system (DAQ). It comprises a signal-digitizing system comprising an oscilloscope with a sampling rate of $8 \mathrm{GS} / \mathrm{s}$ and a bandwidth of $1 \mathrm{GHz}$, which is controlled by a PC/Linux-based computer with a Pentium IV microprocessor at $2.2 \mathrm{GHz}$. The eight signals of the monitor are divided by two signal groups alternately $(2 \times 4$ signals $)$. The four signals of each signal group are sent directly to a signal combiner through coaxial cables with suitable delay lines, which are required to avoid a superposition of the analog signals. The two combined signals out of the signal combiners are directly connected to the digital oscilloscope. Trigger pulses synchronized with the beam are provided to the oscilloscope at a maximum rate of $50 \mathrm{~Hz}$. This rate is applicable for communication between the computer and the oscilloscope through an Ethernet. The computer receives digitized data from the oscilloscope, extracts eight pickup voltages, and calculates the beam positions, the beam charge, and the multipole-moment pulse-by-pulse. It is even possible to observe two bunches separated by $96 \mathrm{~ns}$ in an rf pulse for the simultaneous acceleration of the positron beam. The analyzed data are displayed on any $\mathrm{X}$ terminals through an application program and they are stored in a hard disk.

\section{FEEDBACK CONTROL SYSTEM}

\section{A. Software structure of the data acquisition}

Control software is one of the most important parts for extracting a higher performance from the DAQ system. It must work fast and stably during long-term operation. It is

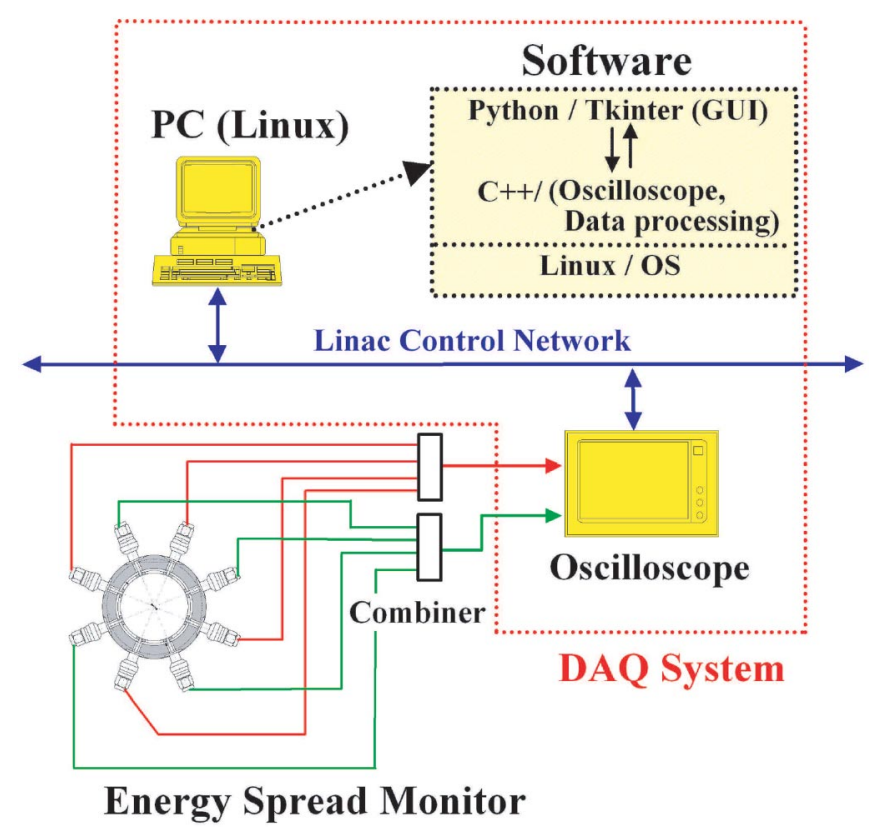

FIG. 2. (Color) Data-acquisition system of the energy-spread monitor. very beneficial to use $\mathrm{C}++$ and python languages for developing such a control software algorithm. The lowlevel layer should deal with the analysis of a signal waveform coming from the monitor electrodes and with the data processing. One cycle of the data acquisition should be completed in less than several milliseconds in order to sufficiently satisfy the acquisition speed of $50 \mathrm{~Hz}$ at maximum. A C ++ compiler language was used for low-level layer development because of its powerful feature with a higher execution speed and a wealth template library for easy development.

The low-level layer transfers the waveform data from the digital oscilloscope to a memory field on a PC via an Ethernet. The peak-to-peak amplitude of the bipolar signal propagated from each electrode is obtained using the measured waveform profile. Then, the PC calculates the beamenergy spread, the beam positions, and the beam charge using calibration coefficients stored in a data file. The dataprocessing algorithm averages 50 successive data, which are written in a local file together with their corresponding one-standard deviations.

To develop the graphical-user-interface (GUI) part, the Python interpreter language was used. The use of the interpreter language instead of compiler languages can accelerate GUI development because the GUI part does not need a higher execution speed very much. The GUI shows time-trace plots of the horizontal and vertical beam positions, the beam-energy spreads, and the beam charges measured by the ESMs in real time during the linac operation.

\section{B. Feedback control algorithm}

The energy-spread feedback control system is indispensable in order to control a beam with higher quality. The main purpose of this feedback control is to suppress the fluctuation of the energy spread caused by the drift of the rf phase of a sub-booster klystron. The drift of the rf phase is mainly originated from the variations of facility environmental parameters (room temperature and cooling-water temperature, etc.) with a relatively long-term period in the klystron gallery. In our feedback control system, the measured energy spread is used as a feedback control value. Its reference target value should be set not at a minimum achievable energy spread but close to it. Since the dependence of the energy spread to the rf phase is described by following a quadratic function [7], it is required to clarify the directional sign of an incremental step of the rf phase in the feedback control algorithm. A simple PID (for proportional, integral, and derivative) control [8] has been adapted to the algorithm for the energy-spread feedback control system. The rf phase of a sub-booster klystron is a feedback actuator in this algorithm. A block diagram of the energy-spread feedback loop is shown in Fig. 3. First of all, the measured energy spread is made a comparison with the reference target value through the feedback algorithm. For 


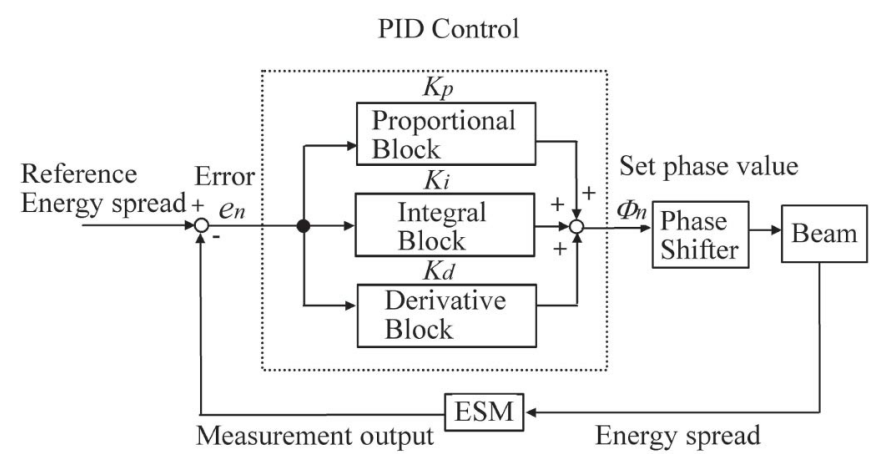

FIG. 3. Block diagram of the energy-spread feedback loop based on a PID algorithm.

the $n$th feedback operation, the rf phase difference $\left(\Delta \phi_{n}\right)$ between the present phase $\left(\phi_{n}\right)$ and the previous phase $\left(\phi_{n-1}\right)$ set value can be written as

$$
\begin{aligned}
\Delta \phi_{n}= & K_{p}\left(e_{n}-e_{n-1}\right)+K_{i} e_{n}+K_{d}\left\{\left(e_{n}-e_{n-1}\right)\right. \\
& \left.-\left(e_{n-1}-e_{n-2}\right)\right\},
\end{aligned}
$$

where $e_{n}$ means the feedback deviation between the measured and reference energy spreads in the $n$th feedback operation; the gains of the proportional, the integral and the derivative terms are denoted by $K_{p}, K_{i}$, and $K_{d}$, respectively. The value set to the actuator is represented by

$$
\phi_{n}=\phi_{n-1}+\Delta \phi_{n}
$$

The feedback control starts to change the rf phase in order to reduce the energy spread towards the reference target value when the feedback deviation increases beyond a threshold energy spread. A beam test was performed in order to adjust the three gains and the threshold energy spread. Constant parameters for the gains $K_{p}$ and $K_{i}$ were set in the feedback algorithm. They were empirically adjusted so as to stabilize the variations of the energy spread with a suitable convergency. The ratio between the gains $K_{p}$ and $K_{i}$ was obtained to be 2.5 . The gain $K_{d}$ was set to zero during the feedback operation, since it was sufficient for the present feedback performance. The threshold energy spread was set to $16 \%$ of the reference target value which corresponded to about twice the measurement error. In our feedback algorithm the sign of the feedback gain $K_{p}$ can be automatically changed when the feedback deviation increases after the next successive three steps so as to avoid any unstable divergence. The upper (lower) limit for a relative increment (decrement) of the rf phase was also set so as to avoid abnormal operation of the feedback control.

\section{BEAM TEST}

\section{A. Beam line}

Figure 4 shows a schematic layout of the beam line of the injector linac and the locations of the beam-feedback control systems. The injector linac delivers four different kinds of beams: single-bunch electron and positron beams to the KEKB storage rings, and a 1-ns pulsed electron beam to two different photon factory storage rings [9], $\mathrm{PF}$ and PF-AR, with injection energies of 2.5 and $3 \mathrm{GeV}$, respectively. They are switched in the beam switch yard at the end of the linac. Here, the beam acceleration and the beam-feedback control scheme for the KEKB operation are briefly described. A single-bunch electron beam can be

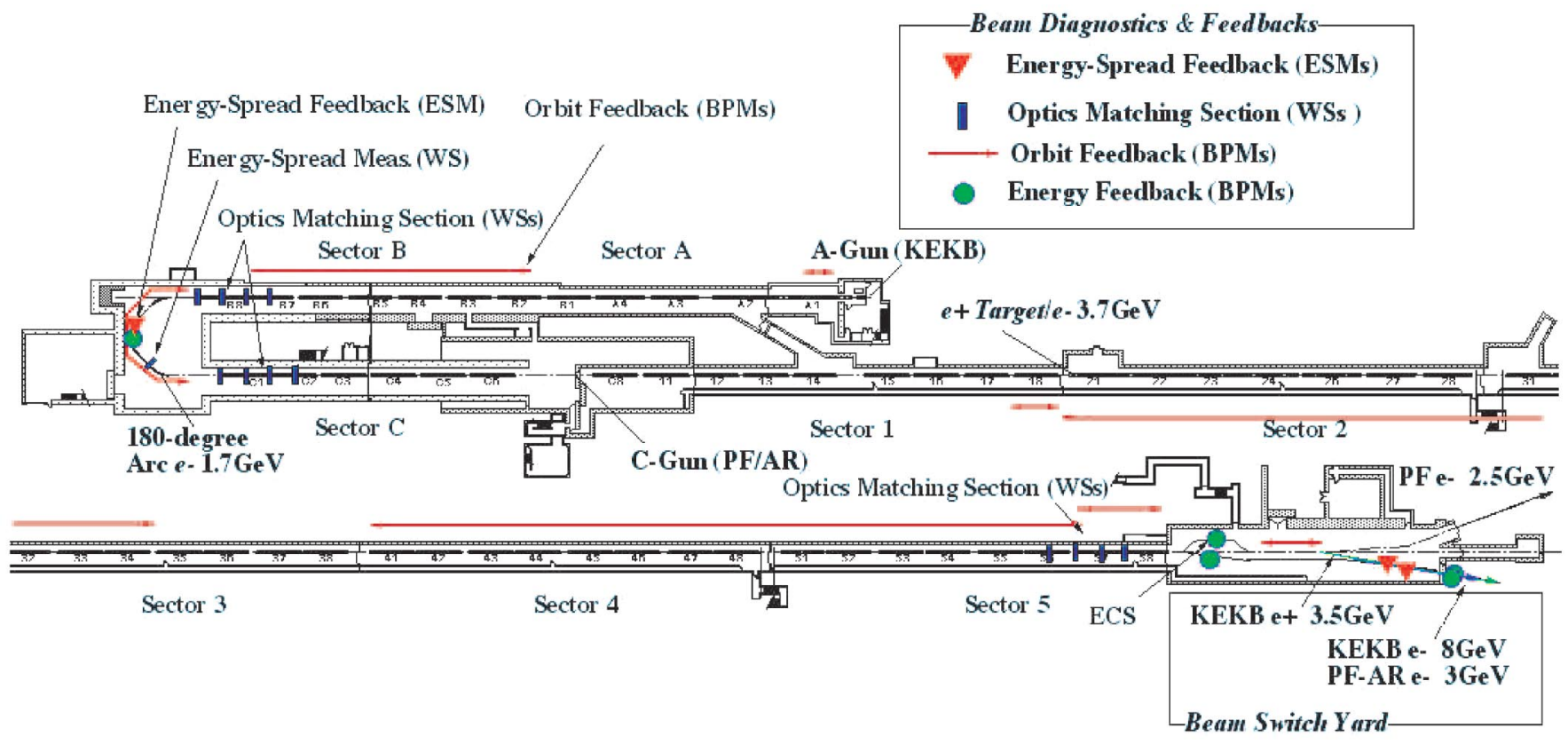

FIG. 4. (Color) Layout of the KEKB electron/positron injector linac and the location of the beam-feedback control systems. 
generated by a new preinjector [10], which comprises two subharmonic bunchers (114 and $571 \mathrm{MHz})$, a prebuncher and a buncher. The electron gun (A-Gun) can generate a beam charge of about $20 \mathrm{nC} /$ pulse with a maximum repetition rate of $50 \mathrm{~Hz}$. For positron production, a singlebunch electron beam greater than $10 \mathrm{nC} /$ bunch can be stably accelerated from the end of the buncher up to a positron-production target installed in sector 2 , where the energy of the primary electron beam is $3.7 \mathrm{GeV}$. The generated positron beam is directly accelerated from the target up to $3.5 \mathrm{GeV}$ at the end of the linac. On the other hand, a single-bunch electron beam is directly accelerated up to $8 \mathrm{GeV}$ at the end of the linac. The nominal beam energy is $1.7 \mathrm{GeV}$ at the $180^{\circ}$ arc, which reverses the beam direction while keeping the achromatic and isochronous conditions for the electron beams in order to preserve the transverse emittances and the time structure of the bunch during its passage through the arc.

Three different kinds of beam-feedback control systems have been implemented along the beam line in order to stabilize the transverse beam orbits, the beam energies, and the energy spreads at different locations. The beam-energy-feedback controls are installed at five locations in a $180^{\circ}$ arc, and the beam switch yard (see Fig. 4). The energy of the $1.7-\mathrm{GeV}$ electron and primary electron beams is stabilized at the $180^{\circ}$ arc. The energy of the $3.5-\mathrm{GeV}$ positron beam is controlled at two locations, an energy compression system (ECS) and the end of the beam switch yard. The energy of the $8-\mathrm{GeV}$ electron beam is also controlled at two locations, the first bending section after the end of the linac and the end of the beam switch yard. The energy-feedback control system has successfully stabilized the beam energies at different locations without any interference with the energy-spread feedback control system during a daily operation. This is due to the use of a control scheme which changes the rf phases at two adjacent high-power klystron stations simultaneously in opposite directions relative to the phase crests with the help of transverse beam-position data measured at a large dispersion section. This control scheme can suppress the increase of the energy spread within $10^{-2}$ of the intrinsic energy spread. The transverse beam orbits along the linac are controlled so as to adjust them to the design orbits with the help of upstream steering magnets through the beamorbit feedback control system [5]. The ESMs and the feedback control system have been newly installed in order to further stabilize the injection beams at three locations with large energy dispersion at the $180^{\circ}$ arc and the end of the beam switch yard. One is for the electron and primary electron beams at the center of the $180^{\circ}$ arc. The others are for electron and positron beams with the energies of 8 and $3.5 \mathrm{GeV}$ at the end of the beam switch yard, respectively. Three optics matching sections using wire scanners (WSs) adjust the transverse phase spaces of the beam at three locations: the end of sector B, the beginning of sector $\mathrm{C}$, and sector 5 .

\section{B. Beam-based calibration}

A beam-based calibration for the ESMs was performed using electron and positron beams under the nominal operation condition in order to calibrate the gain imbalance between the eight electrodes and the alignment errors caused in the installation. The calibration corrects the pickup-signal response and the electrical center positions of the ESM. It was performed by taking mapping data in the transverse plane with respect to the beam axis with upstream horizontal and vertical steering magnets and an upstream quadrupole magnet installed at different locations. Figures 5 show a typical example of the beam-based calibration results obtained for the ESM with the $3.5-\mathrm{GeV}$ positron beam. Figure 5(a) shows that the variations of the quadrupole moment as functions of the horizontal $\left(K_{x}\right)$ and vertical kick angle $\left(K_{y}\right)$ of the upstream steering magnets. The result clearly indicates that the variations of the quadrupole moment have a saddlelike shape over the measured ranges in the transverse plane of the ESM. Figure 5(b) again shows the variations of the quadrupole moment as a function of the horizontal (vertical) beam position fixed at $K_{y}=0\left(K_{x}=0\right)$ along with quadratic fitting functions based on the least-squares fitting procedure. It is also shown that the quadrupole moment depends on the transverse beam positions quadratically in each axis, which agrees well with Eq. (1). The minimum value of the quadrupole moment is given at the fitted saddle point. At this point the difference of the mean squared beam sizes and the offset parameter contribute to the quadrupole moment, since the beam-position dependence in terms of Eq. (1) is cancel out (i.e., $\langle x\rangle=\langle y\rangle$ ). On the other hand, a cancellation of the beam-size dependence can be performed by changing the field strength $(K)$ of the upstream quadrupole magnet. Figure 5(c) shows the result of the quadrupole scan. The minimum value of the quadrupole moment is given at the waist point (i.e., $\left\langle x^{2}\right\rangle=\left\langle y^{2}\right\rangle$ ) determined with a quadratic fitting function based on the least-squares fitting procedure. The offset parameter, finally contributing to the quadrupole moment, was determined to be $g=0.21 \pm 0.1$ for this ESM. The other ESMs were also calibrated based on a similar beam-based calibration.

\section{EXPERIMENTAL RESULTS}

\section{A. Performance of the energy-spread measurement}

A beam test was carried out to investigate the performance of the energy-spread measurement using a primary electron beam for positron production at the $180^{\circ}$ arc with the air conditioner off. It was installed at sector $\mathrm{A}$ in the klystron gallery. Such a condition leads to a large fluctuation of the energy spread because the rf phases of the subbooster klystron and high-power klystrons cannot be entirely controlled due to the variations of a room temperature. Figure 6 shows the results of time traces for the 


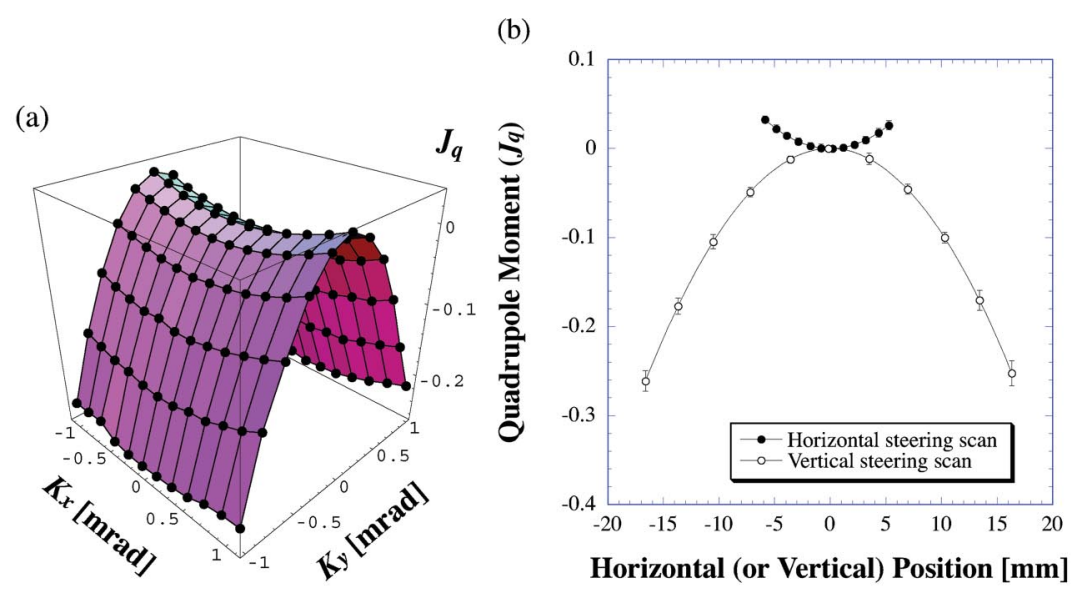

(c)

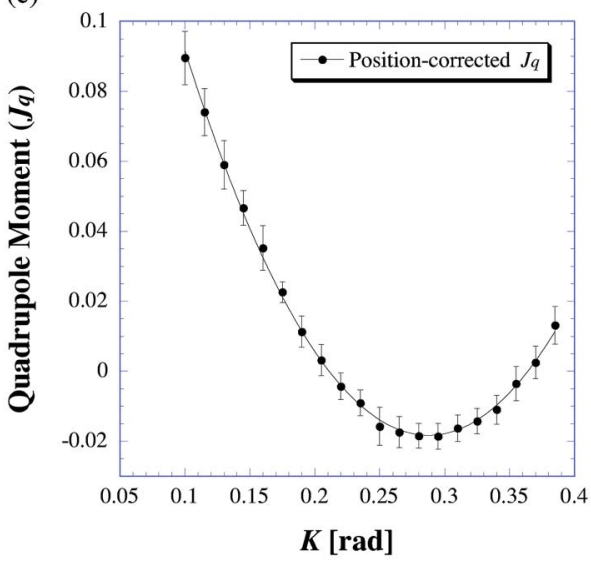

FIG. 5. (Color) Beam-based calibration results of the energy-spread monitor installed at the 3.5-GeV positron beam line. (a) Threedimensional plot in the variations of the quadrupole moment as functions of the kick angles of the upstream horizontal $\left(K_{x}\right)$ and vertical $\left(K_{y}\right)$ steering magnets. (b) Variations of the quadrupole moment as a function of the horizontal (vertical) beam position fixed at $K_{y}=0\left(K_{x}=0\right)$. (c) Variations of the quadrupole moment as a function of the field strength $(K)$ of the upstream quadrupole magnet.

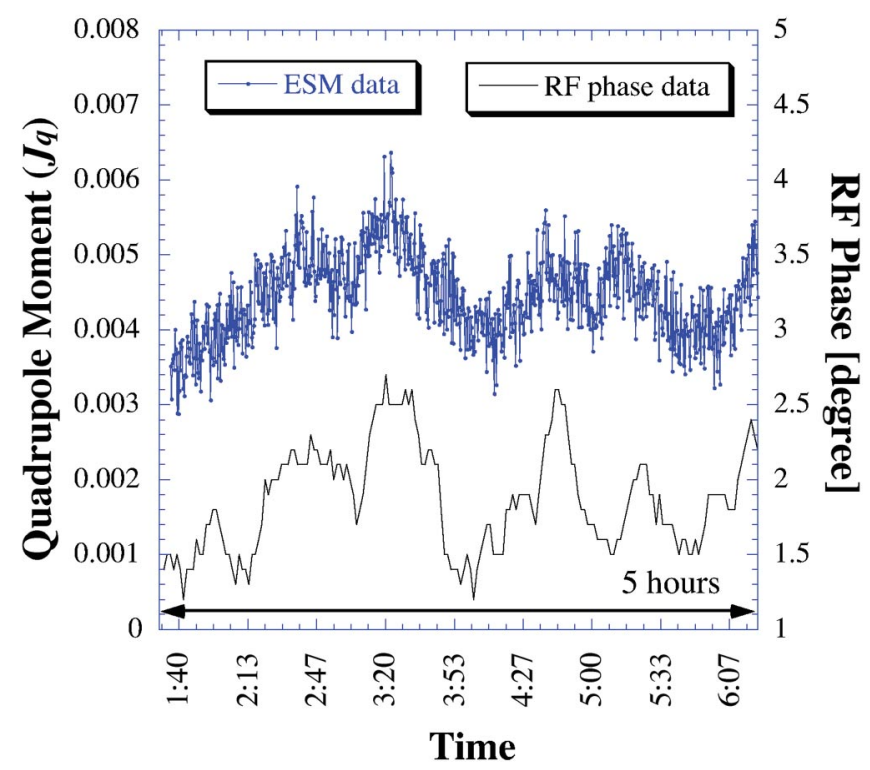

FIG. 6. (Color) Time-trace plots of the energy spread and the rf phase of the sub-booster klystron over $5 \mathrm{~h}$. measured quadrupole moment and the rf phase of the subbooster klystron over five hours. Here, each data point of the quadrupole moment indicates a 50-times average, while they were obtained pulse-by-pulse with a repetition rate of $50 \mathrm{~Hz}$. On the other hand, the rf-phase data were taken every $1.5 \mathrm{~min}$. A linear-correlation analysis [11] was performed to investigate the correlation between the quadrupole moment and the $\mathrm{rf}$ phase. The results show that the linear-correlation coefficient was calculated to be 0.73 where the number of degrees of freedom was 178. The results indicate that the ESM detected the synchronous variations of the energy spread to the rf phase of the subbooster klystron with a good sensitivity.

\section{B. Performance of the energy-spread feedback control}

The performance of the energy-spread feedback control was investigated with two kinds of the beams, the electron beam and the primary electron beam for positron production, at the $180^{\circ}$ arc under the nominal operation condition for the KEKB injection. Figure 7(a) shows time traces of the measured energy spreads for the primary electron beam 
(a)

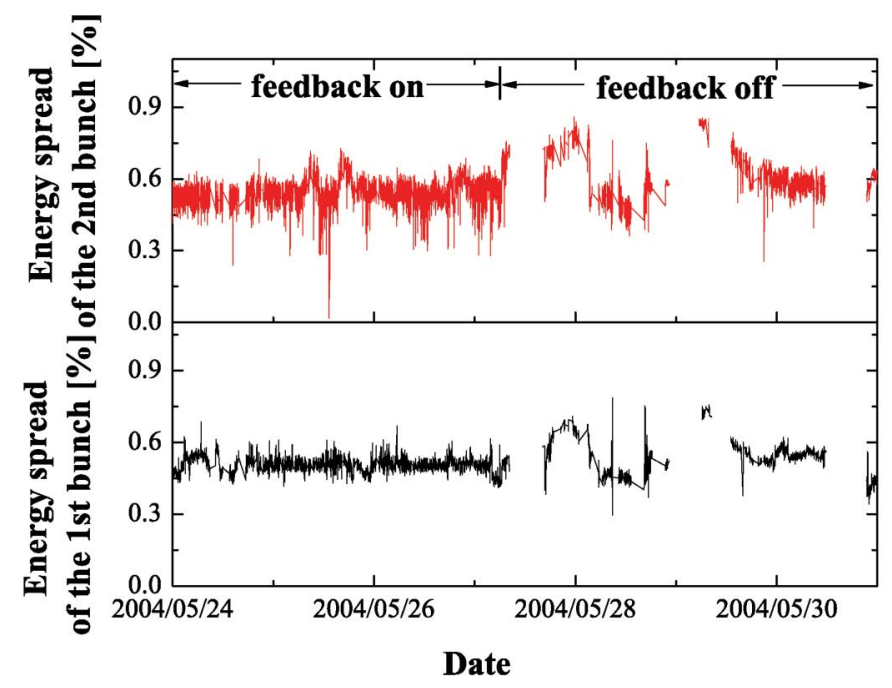

(b)

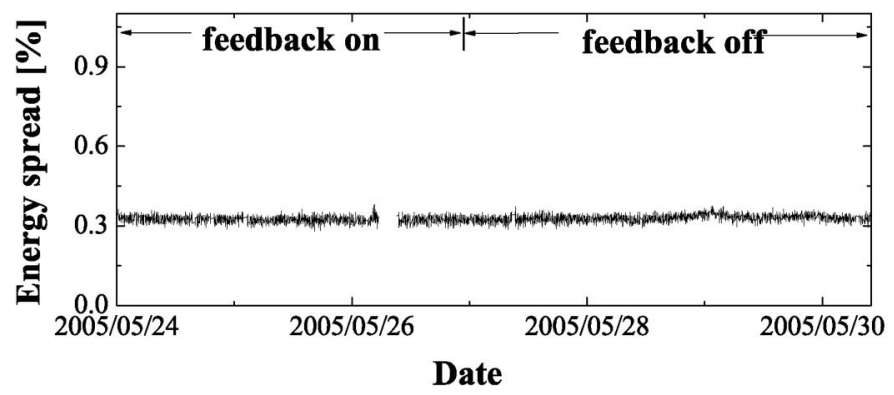

FIG. 7. (Color) Time traces of the energy spreads (a) for the first and second bunches of a high-current primary electron beam in the two-bunch acceleration mode, and (b) for the electron beam, measured at the $180^{\circ}$ arc under the nominal operation condition with the feedback control on and off during seven days.

in the two-bunch acceleration mode with the feedback control on and off over seven days. In this measurement, the other beam-feedback controls, the energy and orbit feedbacks, were also working well without any interference with the energy-spread feedback control. One data point indicates a 50-times average. The results clearly show that the fluctuations of the energy spread were satisfactorily reduced over the time period with the feedback control on. Figure 8 shows a correlation scatter plot and the projected energy-spread distributions during the same days. The solid lines indicate Gaussian fitting functions for the first and second bunches with the feedback control on and off. The energy spreads of the two bunches were stabilized within $0.50 \pm 0.02 \%(0.51 \pm 0.08 \%)$ with the feedback control on (off) for the first bunch, and within $0.55 \pm 0.06 \%(0.62 \pm 0.08 \%)$ with the feedback control on (off) for the second bunch. Here, the error values indicate one-sigma standard deviations of the Gaussian fitting functions. The energy-spread values with the feedback control on are very consistent with the designed energy spread at the $180^{\circ}$ arc. It is worth stressing here that although a same rf phase of the sub-booster klystron was set by following the feedback control in order to simultaneously stabilize the two bunches, the stability of the energy spread for the first bunch was better than that for the second bunch. A relative timing jitter of a grid pulse to the electron gun provided by the trigger-pulse distribution system of the injector linac [12] might generate such an intrinsic energy spread for the second bunch and, however, the measured timing jitter was negligibly small. This might come from the beam-loading effect caused by the first bunch at the preinjector. Figure 7(b) shows a time trace of the measured energy spread for the electron beam at the $180^{\circ}$ arc with the feedback control on and off during the same seven days. We could not find any large differences for the energy spread with the feedback control on and off. The energy spread was $0.33 \pm 0.01 \%$ during the KEKB

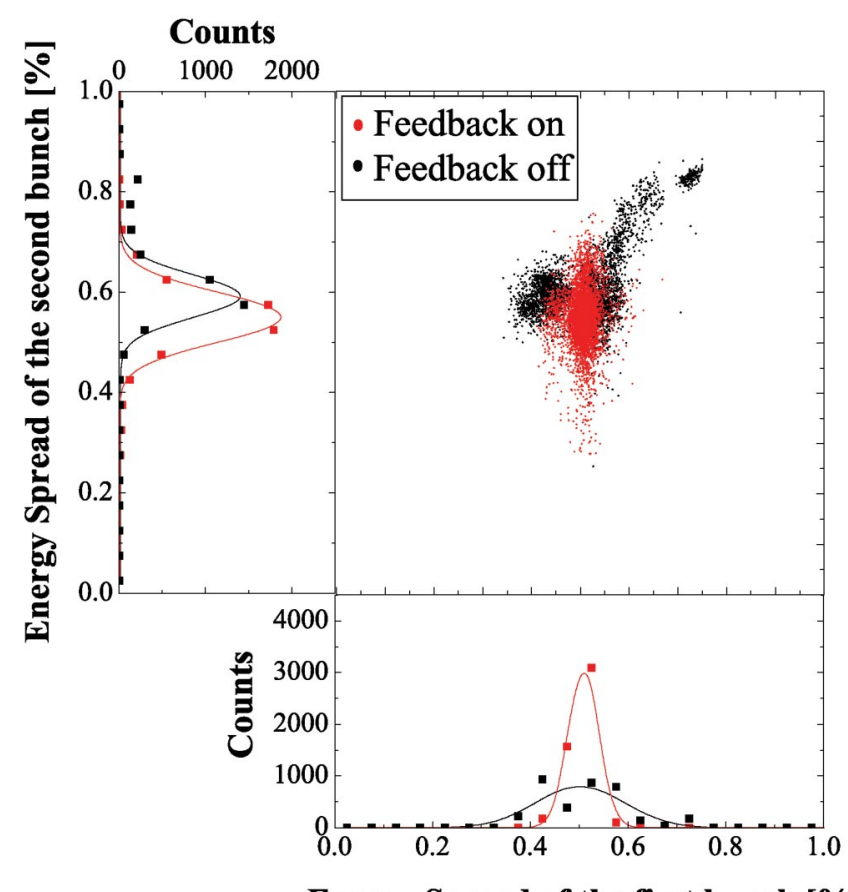

Energy Spread of the first bunch [\%]

FIG. 8. (Color) Correlation scatter plot and the projected energyspread distributions for the first and second bunches of the primary electron beam for positron production in the two-bunch acceleration mode measured at the $180^{\circ}$ arc with the feedback control on and off. 
injection. Thanks to the successful energy spread feedback control at the $180^{\circ}$ arc under the nominal operation condition, the first (second) bunch of the $3.5-\mathrm{GeV}$ positron beam at the beam switch yard has been satisfactorily stabilized within $0.39 \pm 0.01 \%(0.36 \pm 0.03 \%)$ even without the feedback control.

\section{CONCLUSIONS}

An energy spread feedback control system for singlebunch electron and positron beams, and a high-current primary electron beam for positron production, using nondestructive beam-energy-spread monitors with multi-strip-line electrodes, was newly installed and tested in the KEKB electron/positron injector linac. The performance of the energy spread feedback control was investigated and the feedback parameters were tuned in the beam test under the nominal operation condition for KEKB injection. The results show that the energy spread was satisfactorily controlled within $0.50 \pm 0.02 \% \quad(0.55 \pm$ $0.06 \%$ ) for the first (second) bunch of the primary electron beam for positron production at the $180^{\circ}$ arc with the energy-spread feedback control on. The energy spreads for the first (second) bunch of the $3.5-\mathrm{GeV}$ positron beam was stabilized within $0.39 \pm 0.01 \%(0.36 \pm 0.03 \%)$ without the energy-spread feedback control. The energy spread of the electron beam was $0.33 \pm 0.01 \%$ at the $180^{\circ}$ arc. The energy-spread feedback control system has been successfully working in daily operation for the KEKB collider experiment.

\section{ACKNOWLEDGMENTS}

The authors would like to thank the linac operators for their help of this work.
[1] S. Kurokawa and E. Kikutani, Nucl. Instrum. Methods Phys. Res., Sect. A 499, 1 (2003).

[2] I. Abe et al., Nucl. Instrum. Methods Phys. Res., Sect. A 499, 167 (2003).

[3] K. Furukawa, A. Enomoto, N. Kamikubota, T. Kamitani, Y. Ogawa, S. Ohsawa, K. Oide, and T. Suwada, in Proceedings of the International Conference on Accelerator and Large Experimental Physics Control Systems (ICALEPCS'99), Trieste, Italy, 1999 (Elettra, Trieste, 1999), pp. 248-250.

[4] T. Suwada, N. Kamikubota, H. Fukuma, N. Akasaka, and H. Kobayashi, Nucl. Instrum. Methods Phys. Res., Sect. A 440, 307 (2000).

[5] K. Furukawa, N. Kamikubota, T. Suwada, and T. Obata, in Proceedings of the International Conference on Accelerator and Large Experimental Physics Control Systems (ICALEPCS2001), San Jose, CA, USA, 2001, eConf C011127 (2001), pp. 266-268; SLAC Report No. SLAC-R-592.

[6] T. Suwada, Jpn. J. Appl. Phys. 40, 890 (2001).

[7] T. Suwada, M. Satoh, and K. Furukawa, Phys. Rev. ST Accel. Beams 6, 032801 (2003).

[8] J. J. DiStefano III, A. R. Stubberud, and I. J. Williams, Theory and Problems of Feedback and Control Systems (McGraw-Hill, Inc., New York, 1990), 2nd ed., p. 22.

[9] A. Koma, KEK Annual Report 2003, edited by Y. Iwasaki et al., 2004, p. 55.

[10] S. Ohsawa, J.-Y. Choi, Y. Ogawa, A. Enomoto, T. Kamitani, H. Kobayashi, and I. Sato, in Proceedings of the XVIII International LINAC Conference (LINAC'96), CERN, Geneva, Switzerland, 1996 (CERN, Geneva, 1996), pp. 815-817; CERN Report No. 96-07.

[11] P. R. Bevington, Data Reduction and Error Analysis for the Physical Sciences (McGraw-Hill, Inc., New York, 1969), p. 119.

[12] T. Suwada, K. Furukawa, N. Kamikubota, and M. Satoh, Nucl. Instrum. Methods Phys. Res., Sect. A 521, 549 (2004). 